\title{
Session 21.8 - Challenges and Solutions to Light Pollution, RFI and Implementing IAU Resolution 2009 B5
}

\author{
Richard Green \\ Steward Observatory, University of Arizona, 933 N. Cherry Ave., Tucson, AZ 85721 USA \\ email: rgreen@email.arizona.edu
}

\begin{abstract}
The closing session included a panel on the challenge of raising cultural awareness of the negative effects of light pollution and RFI, and a discussion about the means to implement the IAU Resolution on the Right to Starlight. The strongest arguments to the public are that light pollution wastes precious energy and adds greenhouse gases, and that artificial light at night can be damaging to human health and to the natural environment. As astronomers, our community is concerned that the world is blinding itself to the electromagnetic radiation connecting us to the Universe. An outcome of successful advocacy would be to create demand for commercial products that minimize blue light and upward radiation. Implementation of the resolution on the Right to Starlight has multiple aspects. The IAU, through its site protection commission, should provide a clear technical description of "astronomy friendly" lighting and specifications for protection of the near zones around optical observatories. In addition, the commission should provide reference materials for astronomers giving public presentations, provide a forum for those seeking stronger local or national regulation, seek IAU approval for endorsement of protected status of sites and regions, and support the process of gaining UNESCO World Heritage Status for observatories and their regions.
\end{abstract}

Keywords. site protection, light pollution, lighting standards

\section{The Challenge of Changing Cultural Awareness}

The Focus Meeting concluded with a session to address the cultural awareness challenge to stop the increase and reduce light pollution, as well as a plan of action for the IAU through its Site Protection commission. A panel of three passionate advocates for dark skies provided perspective on the challenge of culture change. Colin Henshaw, Elizabeth Griffin, and Audrey Fischer each offered their views on the advantages of quality outdoor lighting that should be most compelling to a thoughtful public.

Uniform lighting at the appropriate level of illumination provides superior nighttime visibility over situations with bright glaring sources producing high illumination and deep shadow. The deep cultural change challenge is in appealing to that rational result to overcome humans' innate fear of the dark and the consequent opinion that brighter is safer. The fear of crime as modern-day threat (as opposed to predatory animals) may be linked to that innate fear of the dark. There is a growing body of evidence, however, that criminal activity is highest during daylight, indoors, or when the moon is nearly full. (See, e.g., http://www .decodedscience.org/full-moons-crime-aka-lunar-effect-real-dealpseudoscience/41881 for a compendium of lunar phase studies.) Collecting the results of research on low light vision, particularly with respect to aging eyes, and how that is best accommodated with outdoor lighting is an important aspect of presenting the facts to the public. 


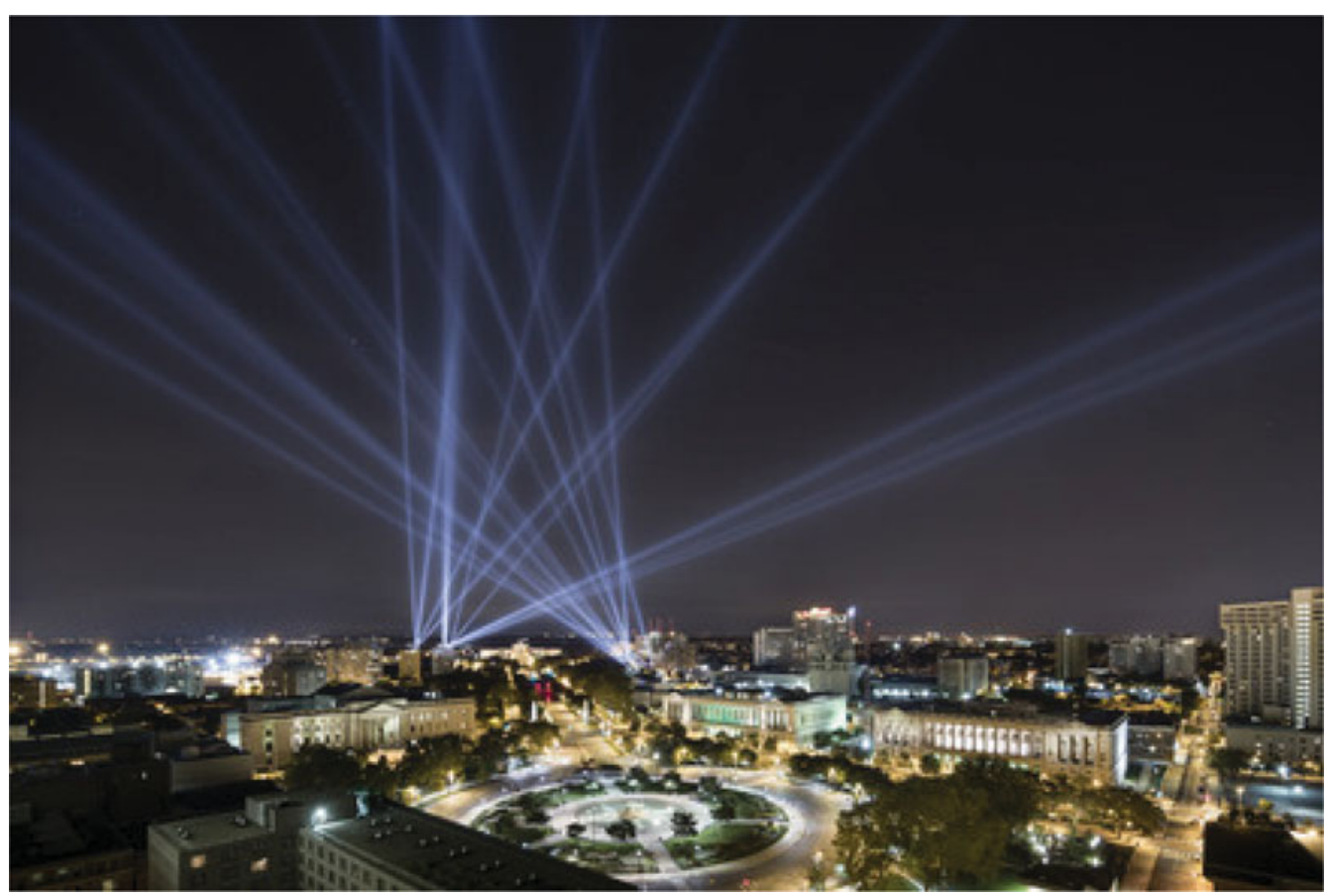

Figure 1. A major cultural challenge is changing the preference for strong artificial urban lighting to design that allows appreciation for darkness and the night sky. Photo of "Open Air Philly" public art display by James Ewing.

The presentation and discussion showed the divergence within the dark sky community on the issue of business motivation. Some feel, based on experience, that manufacturers have a vested interest in creating demand for more and brighter outdoor lighting, that they are willing to link the approach explicitly to nighttime safety, and that their field representatives can even resort to misrepresentation to bring home the sale. A broader view is that manufacturers have a vested interest in increasing sales, which they will do by both creating and trying to meet customer demand. Therefore, educating the public with the goal of creating demand for quality outdoor lighting can actually drive the market in a positive direction. Presentations by the lighting design engineers earlier in the session illustrated the growing availability of spectrally controlled LEDs and full cut-off fixturing in response to demand.

Light trespass is the most common complaint relative to outdoor lighting. Lack of directional control can send light from streetlights or outdoor advertising directly into residential windows, generating legitimate complaints. There is a wide range of response by localities and commercial interests to such complaints from region to region. A proposed goal is for the IAU and those working for the principle of the right to starlight to help assert the rights of "quiet enjoyment" for residents. Light trespass should be made comparable to noise trespass or any other external infringement on the living space of individuals. In many regions, particularly in dense urban areas, there is much work to be done to rebalance the approach to lighting, with protection against trespass and the legal means to enforce that protection as a critical beginning. The major cultural challenge is overcoming today's urban misconception: the belief that they need to sacrifice their starry night sky for urban light, as illustrated in Figure 1.

The presenters pointed out that stretches of highway without significant cross-traffic do not require external illumination for safe operation of vehicles. Headlamps are 
sufficient, and in the case of multiple lane roads with the two directions of traffic in close proximity, light baffling barricades or fencing can reduce the glare from oncoming headlights. Technological development holds real promise for motion-activated illumination at critical areas with cross-traffic or pedestrian activity.

In the end, the astronomical need for tighter control of artificial light at night may be just a corollary benefit from changes brought about by the growing realization that human health can be adversely impacted. Blue light inhibits production of melatonin; the system is presumably balanced to inhibit production during the day, while allowing production during the dark hours of night. Artificial light rich in blue light content is demonstrated to change the balance of that production rate. Since melatonin can play a role in suppressing cancerous growths, blue light at night can contribute to increased health risk. To the extent that artificial lighting disrupts the balance between insects and their predators like birds and bats, an increase in artificial lighting can increase the incidence of disease vectors, such as mosquitoes, which carry highly infectious and debilitating diseases in the developing world.

Given the mounting pressures on local and municipal budgets, economic arguments in favor of minimizing wasted light should be gaining traction. One estimate is that some $45 \%$ of outdoor lighting goes non-productively to direct uplight and overlighting. In aggregate, that amounts to $52.2 \mathrm{TWH}$ per year in the US, or some 38 million tons of $\mathrm{CO}_{2}$, a strong contribution to unwanted greenhouse gas accumulation.

Obviously there is an environmental impact associated with the wasted energy of poor quality outdoor lighting. Another perspective on the way to approach the cultural change necessary for the public to generate the demand for reduced and higher quality outdoor lighting is by reconsidering the three R's of the environmental movement. In addition to reduce, re-use, recycle, a desirable thought framework is that we have a moral duty to respect the planet and the wellbeing of humans and all biological systems. Those three R's are Respect, Responsibility, and Right. By intellectually disentangling want from need, individuals could adopt an approach of lower demand for energy use in general, and lower tolerance for wasteful excesses in outdoor lighting in particular.

\section{Implementation of IAU Resolution 2009 B5 - The Right to Starlight}

All of these considerations enter into the approach that astronomers and the IAU should be taking to address the goals of IAU Resolution 2009 B5, "In Defense of the Night and the Right to Starlight". It speaks to the night sky as an inspiration, to its scientific and cultural values, to the view of the night sky getting worse, to the need to educate the public, to the need to use intelligent lighting, and to astro-tourism.

Key Excerpts from the Resolution:

An unpolluted night sky that allows the enjoyment and contemplation of the firmament should be considered a fundamental socio-cultural and environmental right, and that the progressive degradation of the night sky should be regarded as a fundamental loss; IAU members [should] be encouraged to take all necessary measures to involve the parties related to skyscape protection in raising public awareness of the educational, scientific, cultural, health and recreational importance of preserving access to an unpolluted night sky for all humankind.

Protection of the astronomical quality of areas suitable for scientific observation of the Universe should be taken into account when developing and evaluating national and international scientific and environmental policies, with due regard to local cultural and natural values. 
Presentation and discussion of the last session centered around the general means to accomplish these objectives and specific steps that the new IAU Commission C.B7 could take to empower astronomers to address the issues successfully.

Five general approaches were agreed to be appropriate to this worldwide task. One is engagement of more of the astronomy community in including this message in their public outreach activities. Having the IAU Commission website provide one or two effective slides could be very powerful. Second would be provision of vision and background statements, with supporting material, to those astronomers who are prepared to be more engaged in educating the public. This is where the International Dark Sky Association (IDA) can be a prime source, because of their strong approach to implementing sky protection goals.

The next general approach is working to create demand for the new products available to support astronomer goals of full cut-off and limited spectral pollution. This area is where Commission 50's highly beneficial and productive engagement with the International Commission on Illumination, CIE (http://cie.co.at/), is worth amplifying to keep the positive interaction at a high level. Since the last GA, such products are now commercially available, and as discussed above, the lighting industry will sell whatever consumers demand.

A key element is helping those astronomers who are engaged with local, national, and international authorities to protect astronomical sites. Past efforts of the previous Commission 50 and the World Heritage Working Group under Division C are beginning to pay off to get sites into the World Heritage category for protection. IAU can also provide materials and advice supporting astronomers who are engaging local and national authorities for lighting regulation to protect natural, historic, and ordinary local areas from light pollution encroachment.

The key question is how to turn those general approaches into a practical implementation plan with goals and milestones that can be tracked. Following are the specific programs that were discussed as an implementation strategy.

The imprimatur of the IAU will be particularly valuable in enunciating a clear technical standard for "astronomy friendly" lighting, including full cutoff, spectral management, and minimizing lumens required for the task.

There was unanimous agreement that the highest priority was full cutoff, with no light emitted directly above horizontal. Gaining adoption of that approach is an uphill battle, both with those who develop standards and those who implement lighting designs. The former group is dominated by engineers who deal with specular reflection and are much less familiar with the radiative transfer approach that demonstrates clearly the strong negative impact of direct upward radiation. The latter are faced with budgetary limitations that tempt them to limit the height of poles and to tilt the luminaires to cover more area.

The issue of spectral management requires sound technical advice. The availability of new LED products with low correlated color temperature (CCT), built-in full cutoff filters, and even narrow-band emission gives a number of options for choices with very low blue light content. As was shown in the presentations by the lighting engineers summarized in FM21.1, a single number like CCT is not adequate to describe color rendition or blue light suppression. Development of a clear guideline or set of acceptable technical choices is a priority goal for the new Commission with the aid of its technical members.

Incorporating the latest research on safety and night vision will be critical for developing industry standards that move in the direction of lower illumination appropriate for usage and area, as opposed to illumination levels with large margin. Curfews and 


\section{Moonlight and 2200K are much, much more similar \\ LED is not "natural"}
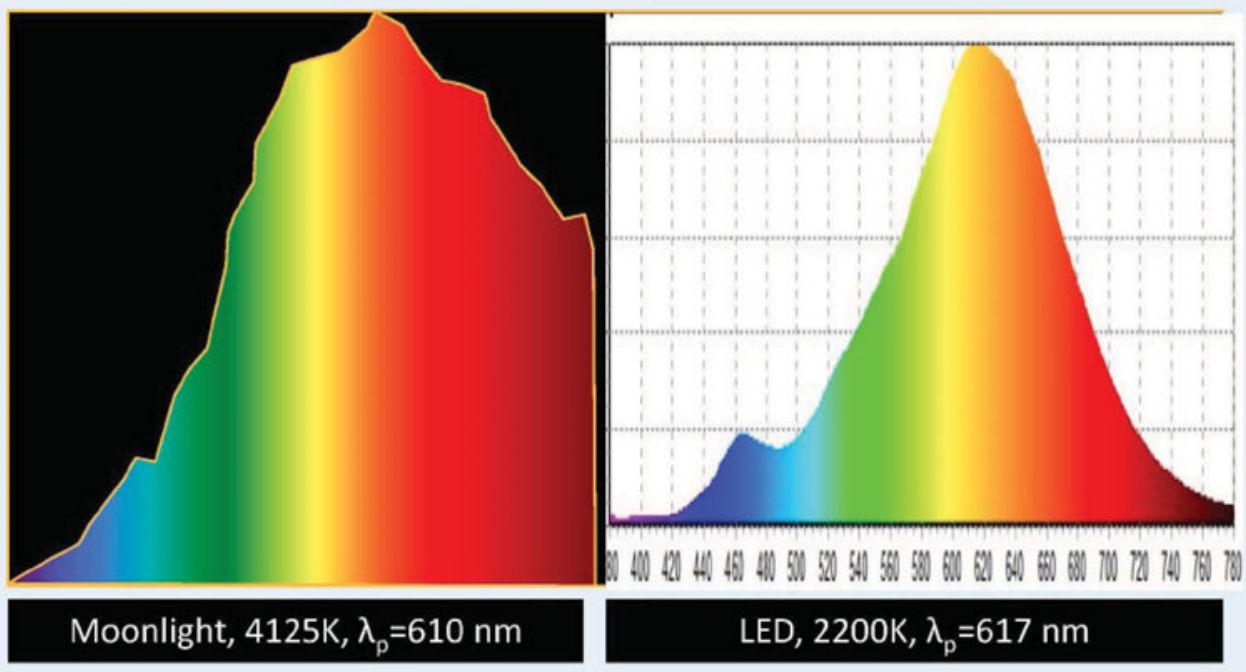

Figure 2. Correlated Color Temperature dervied from fitting a blackbody curve is not an adequate measure for characterizing the color distribution or blue light content of an artificial source like an LED. IAU through its site protection commission must develop clear recommendations for "astronomy friendly" lighting. Figure from talk by Monrad, as summarized in FM21.1

motion activation can also be part of the IAU recommendation for appropriate lighting. The challenge for this set of recommendations will be to find dark sky friendly levels that do not seem to be in contradiction with current engineering standards.

A function unique to IAU activities is to detail the standards for near zone professional observatory site protection. These would entail extremely low lumens levels per hectare and the employment of narrow-band sources, even if energy savings were not as great as with broader-band LEDs. Of course, such a reference standard would need to be modified in individual cases (e.g., existing nearby towns with commercial districts for which rural low illumination levels would be inappropriate). Nevertheless, having a recommended template as a starting point would be of value for site preservation efforts around the world.

To support the general approach of encouraging astronomers to include dark skies issues in their public lectures and talks with local policy makers, the IAU Commission should develop (i.e., write a bit and collect a lot of) information to comprise an IAU dark skies "standard" outreach package. The task is then to launch an outreach effort to professional colleagues, to get them beyond the opinion that site protection and dark sky preservation in general is a job required only of observatory directors and their designated staff.

The Cornerstone programs developed for the International Year of Light (IYL) were chosen because of their broad international impact and appeal. A very near-term goal will be to assure that IYL Cosmic Light projects and other successful IYL astronomy programs achieve sustainable status. The effort-based part will be to continue to publicize availability through the Commission website. Should some funding be needed to put a 
program on more solid footing, the Commission will explore with the project PIs options like crowd funding or continuing commercial sponsorship.

To take advantage of the opportunity to influence international lighting standards, we intend to re-energize active interaction with the CIE, with formal IAU liaison and practical means of participation in their technical committees defining standards. CIE has welcomed IAU involvement. With their worldwide activities and meetings, support will require a small network of technically conversant Commission members who can cover meetings on multiple continents.

A key aspect of the top-down approach to control light pollution is the drafting, implementation, and updating of outdoor lighting standards and regulations in local and regional legal codes. The Commission can provide a forum for voluntary coordination and cooperation among those working to protect key astronomical sites, as well as those concerned with preserving sky quality in natural protected regions or even urban settings. An obvious activity is exchange of information on techniques for getting local and national lighting ordinances passed.

Another way in which the reputation of the IAU can be used to good purpose is in the production of IAU letters of endorsement to relevant entities attesting to the astronomical value of a site. Observatories or regions seeking special designation, for example with the IDA, seek such letters, and they have proven to have real value in such activities. An obvious extension would be endorsement for a proposal to a locality or other government agency to retrofit lighting that would significantly improve dark sky conditions near an astronomical site.

The full scope of the IAU Resolution provides a broad charge to astronomers for dark sky preservation. In that context, the Commission should consider ways to encourage protection of natural and historic sites against light pollution encroachment. As presented in this focus meeting, the US National Park Service has been proactive in protecting dark skies as part of the unique environments of their Parks. Public viewing through small telescopes has become a major visitor attraction in some of these parks. Active encouragement of similar approaches on a worldwide basis is an obvious role for astronomers according to the Resolution.

The Site Protection Commission must continue its support of the World Heritage Working Group and UNESCO with ongoing and new projects. The immediate goal is declaration of observatory sites in Chile, and possibly in Spain and Hawaii, as World Heritage sites. The Commission can serve an advisory role in the preparation of the application materials to present the sites as uniquely well suited for astronomy in terms of their dark sky and other atmospheric qualities. Its experts (although a different set) can similarly advise UNESCO when they come to evaluate the proposal.

Finally, the Commission must define a practical means of partnering with the IDA to leverage international outreach for both organizations. Their reference and presentation material is of high value, and their international chapters can provide some local language support. Direct association with the IAU can strengthen their international reach, and help both organizations improve their support base for dark sky protection activities.

\section{Afterword}

This ambitious agenda was endorsed by the dedicated and passionate group participating in the Focus Meeting. The ongoing challenge is to focus the volunteer effort to best effect and grow the level of participation dramatically. Only with the conscious effort of a significant fraction of the astronomers in the IAU can we reasonably aspire to tackle the global goal of universal acknowledgement of the right to a dark night sky. 